Fertility regulation during human lactation

\title{
STUDIES ON LACTATION AND CONTRACEPTION IN WHO'S RESEARCH PROGRAMME
}

\author{
R. M. BERNARD \\ Human Reproduction Unit, World Health Organization, Geneva
}

\section{Introduction}

Although steroid contraceptives have been in widespread use for many years, there still appears to be considerable uncertainty in the minds of many clinicians and family planning programme decision-makers regarding their prescription to nursing mothers. This uncertainty appears to be reflected in replies to a questionnaire sent by the Human Reproduction unit of WHO to scientists in its network of Collaborating Centres for Clinical Research in Human Reproduction regarding the current status of the use of steroid contraceptives by nursing mothers in different national settings. Although the information was, of necessity, in some cases imprecise, the following impressions were gained about eighteen countries: (Australia, Canada, Chile, Egypt, Holland, Hungary, India, Mexico, Nigeria, Philippines, Singapore, Sri Lanka, Sweden, Thailand, Turkey, United Kingdom, USA, Zambia).

Government policies seem to vary from unqualified or qualified approval or recommendation to disapproval, and not surprisingly the practice in sectors other than governmental tends to be influenced by the given policy. In the absence of specific government policy, a situation apparently applying in approximately half of these countries, the decision seems to be left to the discretion of individual practitioners. Existing policies or practices are by no means uniform and vary in respect of different preparations, their availability and the timing of first administration after delivery, and the importance placed on breast-feeding, which in developing countries is almost the only way to ensure adequate nutrition of the infant. Such are the variables involved, including patient preference for a given method, it is difficult to estimate on a global scale the extent to which steroid contraceptives are prescribed to nursing mothers, and more information is needed on this point.

It is now generally accepted that the post-partum phase represents one particular phase when women may most readily be motivated to accept family planning advice. Some will accept an IUD, others will prefer a traditional method, and others will prefer a steroid contraceptive and be reluctant to use any other method.

It has been postulated that the recommendation, based upon potentially adverse effects, to withhold steroid contraceptive prescription until lactation is fully established or until 3 or more months post-partum, has led to the loss of many 
potential acceptors, because, following the break in continuity of care these women frequently fail to return. Whilst this may be the case, if a nursing mother using steroid contraception has any suppression of lactation it may lead to impaired infant growth and increased risk of morbidity associated with early supplementary feeding. To remedy matters she may decide to discontinue its use, but will then expose herself to the risk of further conception unless she uses an alternative form of reliable contraception. On the other hand, if her main concern is to avoid conception she may elect to continue with its use at the expense of lactation and wean her infant prematurely, unaware of the risks associated with unhygienic or inadequate artificial feeding.

There is still insufficient scientific knowledge upon which to draw definitive and universally applicable conclusions concerning which steroid contraceptives may be safely prescribed to breast-feeding mothers, and in what dosage and how soon after delivery. There are two fundamental questions which have not been resolved to date with absolute certainty. First, do contraceptive steroids individually or in combination have any adverse effect on lactational performance, measured by the quantity and quality of milk supply and the duration of lactation? Second, to what extent if any are these steroids in biologically active form transferred via the milk to the infant, and what is the significance of such transfer?

The need for further research to clarify uncertainty regarding these issues has been repeatedly identified during the last few years (Chopra, 1972; IPPF, 1976; Buchanan, 1975; Thomson, Hytten \& Black, 1975; WHO, 1975). Dissemination of the knowledge gained would not only enable physicians to advise their nursing patients with confidence on the effects of steroid contraceptives if used during lactation, but should provide clear guidance to decision makers in the development of post-partum strategies in their family planning programmes.

The World Health Organization is making efforts to respond to this recognized need through its Expanded Programme of Research, Development and Research Training in Human Reproduction.

\section{Influence of steroid contraceptives on lactational performance}

A study of the published research to date reveals the problems which faced those who have attempted to draw conclusions from reviews of the pertinent literature (Chopra, 1972: Buchanan, 1975; Thomson et al., 1975; Vorherr, 1973). In summary, it may be said that the interpretation of the results from the many studies undertaken is difficult for many reasons - variations in the type and dose of preparations used, in the timing of first administration, and in the frequency and duration of observations; variations in the methodology used to measure lactational performance, e.g. maternal subjective impressions and retrospective estimates of the duration of lactation, even including that following previous pregnancies, have been used in some studies; variations in, or absence of information on, the precise methodology used to quantify milk volume; and many studies do not appear to have taken into consideration such variables as infant birth weight and maturity, infant or maternal morbidity, parity and maternal age. In many studies control subjects have been omitted and in comparing the effect of different pills the use of a 
double-blind packaging approach, which many would consider essential, is not specifically mentioned.

Thus it becomes difficult to draw conclusions. Nevertheless, despite these reservations it seems clear that certain generalizations can be made. The majority of published studies imply that combination pills containing high doses of oestrogen, and even in certain studies implicating those containing $50 \mu \mathrm{g}$, adversely affect lactational performance, but to what extent this effect is significant is very open to question. Indeed in a recent editorial article in the Lancet (Anon, 1976), attention is drawn to the conclusion of Thomson et al. (1975), following their wide review, that where efficient contraception is required, low-dose oestrogen-containing oral preparations present no serious threat to continued breast-feeding. The effects most frequently reported, measured either subjectively or objectively, are a decrease in milk volume requiring early or additional supplementation, and a decrease in the duration of lactation in some cases with premature cessation.

No studies are as yet known to have been published on the use of new low-dose oestrogen combination pills, i.e. those containing $30 \mu \mathrm{g}$ of oestrogen, towards the use of which there appears to be a trend in some countries.

On the other hand, the majority of studies concerned with the use of progestogen only oral preparations have shown no detrimental effect on lactation, nor have those investigating the use of injectable preparations, which have been reported by some investigators even to enhance lactation.

The evidence from those studies which sought to determine the effect of combination or progestogen-only oral steroid contraceptives and injectable preparations on the composition of human milk is conflicting and difficult to interpret-perhaps due to the variations in the preparations studied, the timing of their administration, the duration of observations, and in assay techniques.

Studies in this whole field are acknowledged to be difficult to conduct, but against this overall background of doubt and uncertainty arising from multiple individual studies one approach appears necessary - the conduct of a collaborative study in several centres from both developed and developing countries to compare prospectively the effects of a limited number of different steroid preparations on the lactational performance of nursing mothers.

Such a study entails the elimination of as many variables as possible by, e.g. (1) pre-treatment observations at fixed times post-partum, (2) the commencement of contraceptive therapy at a fixed time post-partum in the study groups, (3) the use of control groups, e.g. those who elect to take no contraceptive measures and who are not concerned whether they become pregnant or not, and those who elect to use a non-medicated IUD, (4) matching as far as possible subjects between groups for maternal age, socio-economic class, parity, infant birth weight and maturity, within specified ranges, (5) adopting a standardized approach to the collection of milk to assess output at prescribed intervals over a prescribed period of time, and (6) the use of standard milk sampling and assay techniques for the assessment of composition.

Since there is a suggestion that even those combination pills containing only $50 \mu \mathrm{g}$ of oestrogen may have an adverse effect on lactation, however inconclusive the evidence, it would now seem more prudent to compare the effects of an oestro- 
gen combination pill containing only $30 \mu \mathrm{g}$ oestrogen with that of a pill containing only the same type of progestogen used in the combination formula, e.g. d-norgestrel + ethinyl oestradiol versus d-norgestrel only. Such pills should be prepared with an identical appearance and packaged to ensure double-blind comparison. Although obviously it would be impossible to compare in addition the effects of injectables on a double-blind comparative basis the inclusion of groups given either DMPA or NET-oenanthate would be highly desirable.

Careful monitoring of both infant and maternal progress would be mandatory.

If such a project, a protocol relating to which is at present in the final stages of review within WHO, could be implemented successfully, it should provide much of the information needed for the solution of the first question.

\section{Transfer of contraceptive steroid hormones/metabolites and their fate/ significance as far as the infant is concerned}

If steroid contraceptives are prescribed post-partum to nursing mothers, it is conceivable that their offspring whose detoxicating mechanisms may not be fully developed would be exposed to these steroids or their active metabolites if they were to appear in the maternal milk.

No studies have yet been performed which clearly demonstrate the significance of any such possible transfer via human milk as far as the infant is concerned, especially if steroids are prescribed to these mothers over prolonged periods. Vorherr (1973) in his review of current developments in contraception after abortion and post-partum concluded that in the nurslings of mothers ingesting the pill no adverse effects in regard to bone growth, genital development and impaired fertility later in adulthood can be substantiated, and doubted whether sex hormones as contained in present pill preparations are transferred via the breast milk into the system of the nursling in sufficient amounts to cause accelerated bone maturation or any other adverse effects.

Work in this whole field has been hampered by the difficulties encountered in developing reliable assay techniques for the study of contraceptive steroids and their metabolites in milk.

It is difficult to draw any firm conclusions from the very few preliminary studies which have been published relating to human research in this field, and which have involved the use of radioactive isotope labelled steroids, of necessity given to lactating but non-nursing mothers (Laumas, Malkani \& Bhatnagar, 1967; Van der Molen, Hart \& Wijmenga, 1969; Pincus, Bialy \& Layne, 1966; Wijmenga \& Van der Molen, 1969). In three of the studies it was specifically stated that the study was performed in the immediate post-partum period and lactation was not established by suckling. Considerable variation occurred in the volumes of milk obtained by breast pumping but, in general, volumes were low. Conclusions had to be based upon the percentage of radioactivity recovered from the milk, but not upon the percentage of biologically active steroid or inactive metabolites. These percentages varied from 0.0002 to $0.013 \%$ in the cases in whom labelled oestrogens were given and 0.0002 to $1.5 \%$ in the cases in whom labelled gestagens were used. Thus at this stage any thoughts on implications must be purely specu- 
lative, and more work is needed, particularly in view of the trend towards the use of lower dose and/or different preparations even in non-lactating women.

The difficulties of extrapolating animal findings to the human are well known. Nonetheless, toxicological studies of which we are aware have shown no evidence of any effect on the offspring of nursing rhesus monkeys given up to $100 \mu \mathrm{g}$ ethinyl oestradiol $/ \mathrm{kg}$ body weight daily for 12 weeks. Vorherr (1973) in his animal experiments gave lactating post-partum mother rats oral oestrogen-progestogen doses comparable on a weight basis to those given in the pill. Neither these doses nor those up to five-fold the corresponding human oral contraceptive doses led to impairment of the suckling pups' weight gain, maturation or reproductive function, or to the development of cancer later in their lives.

Despite the progress in development of reliable techniques of steroid assay, such are the problems of interpretation that even in the light of present day knowledge it may take many years of investigation to reach definitive conclusions with respect to humans.

Towards this end, WHO is planning to support research designed to study the transfer of commonly used progestogens and oestrogens in oral contraceptive preparations and progestogens in injectable preparations and to quantify and to identify the nature of whatever form of these steroids may be transferred, be it biologically active or inactive, as a first step towards the solution of the second question.

\section{Return of fertility during lactation}

There is sufficient evidence in the literature to show that lactation gives some measure of protection against conception, but the mechanism whereby this is achieved remains to be clarified. Although this protection may be substantial during the phase of lactational amenorrhoea, unacceptably high rates of pregnancy (up to $10 \%$ ) during this phase have been reported, with a marked increase following the return of menstruation but before weaning (Buchanan, 1975). The duration of protection can thus only be measured in broad terms and cannot be predicted with certainty. If a simple technique could be devised which would enable a nursing mother to determine with some exactitude when she is first at risk of becoming pregnant this would obviously represent a major advance.

The WHO Task Force (WHO, 1976) primarily concerned with research concerning methods for the determination of the fertile period is accumulating data from several centres on the correlation between ovulation, as determined by direct observation and plasma levels of steroids, and gonadotrophins to identify those parameters which could be used as reference points to judge the reliability of methods developed for the prediction of the fertile period which are suitable for home use. Efforts are being made to develop assay kits which would allow women to predict and to detect the occurrence of ovulation. Radioimmune assays have been developed for various urinary oestrogen and progesterone metabolites which could possibly form the basis of such 'do it yourself kits', if continuing research confirms specific relationships with the occurrence of ovulation. The scientists in 
this Task Force are in addition developing and using devices from the fields of medical physics and bioengineering to investigate changes in vaginal temperature, heat flux, blood volume, colour and secretions and in biopotential associated with ovulation.

The promoters of fertility regulation based on periodic abstinence believe that women can be taught through awareness of changes in their cervical mucus, with or without monitoring changes in basal body temperature, how to recognize the onset of the fertile period, regardless of their reproductive cyclic status. The Task Force is currently investigating on a multi-centre basis the use-efficacy of these methods of fertility regulation in women with normal ovulatory cycles. Should these methods prove reliable further consideration will be given to the exploration of their use during lactation as a means whereby the timing of ovulation, and thus the need for contraceptive measures or abstinence, can be identified.

\section{Discussion}

Thomson: How do you propose to ensure the regularity of taking the contraceptives? I take it that these people will not just be given packets of the appropriate hormonal preparation and left to it? There will have to be some kind of supervision of the regularity of taking them and so on, especially in relatively unsophisticated communities.

Bernard: The scientists with whom it would be proposed to collaborate are fully aware of the need to monitor the usage of contraceptives and the importance of such control. It is proposed that subjects be seen twice before the start of contraceptive therapy at six weeks post-partum when there can be no doubt that lactation is fully established. Thereafter mother and child would be seen at monthly intervals for six months at the concerned hospital centre by physicians, and intermediate visits would be made to the home by a health visitor, to ensure essential medicare and to check on the regular use of the given contraceptive. The women would enter the study after careful explanation of its aims and particularly for the need for regular pill taking.

Robyn: Is any comparison being made between what lactation can achieve, in terms of contraception, and what can be achieved by the modern methods taking into consideration their acceptability which may be very low? Is there any possibility of making such a comparison in your study?

Bernard: It is not intended to make such a comparison. Since only a limited number of subjects would be observed, including non-contracepting controls, over a very limited period of time, efforts to assess contraceptive efficacy would probably not be meaningful under these circumstances.

Thomson: You talked about a controlled study, then about women electing to take part. Do you think that the effects of self-selection can be overcome by matching? A randomized trial would be ideal, but is scarcely feasible.

Bernard: I agree that such a randomized trial would not be feasible. Randomization only to the use of different contraceptive pills can be achieved but not to placebo pills which are ineffective contraceptives. The woman must be allowed to use the method of contraception of her choice. Within such a study as far as 
matching is concerned every effort would be made to eliminate as many variables as possible.

Mosley: Would it be considered ethically right to take a group of IUD acceptors who would not object to taking the pill and to ask them to take two or three cycles of the pill, including a placebo preparation? This would get around the problem of controls.

Bernard: Ethical questions always require considerable reflection. If it were considered ethically right then it may well be that your suggestion would get around the problem of controls.

Mosley: It would be possible to assign randomly the preparations, including the placebo.

Thomson: We cannot exclude possibly awkward interaction effects.

Mosley: I agree. But we might wonder whether we should neglect to look for the effects of an extremely important variable because of the possibility of some potential interaction effect which is unknown.

Thomson: In effect, 'Let not the best be the enemy of the good'.

Gray: The objective of the study is purely comparative. About the controls, and the different study strategies that can be adopted, each woman will be her own internal control, insofar as changes over time are being measured. There would be ethical problems with the use of a placebo, but I doubt that placebo effects would be sufficient to alter the findings. If there are pure placebo effects related, say, to the use of the pill, these should be seen in the oestrogen-containing group as well as in the progestogen only group. I do not think a cross-over design would be practicable, because of the changes in lactation over time which would make such a design extremely complex.

Tyson: Will there be any limits on the number of times that the woman breastfeeds her child during the study?

Gray: No-the objective is to try, as far as possible, to eliminate the effects of mother-child interaction. If there is an effect of the steroids, it is presumably an effect on the capacity of the breast to produce milk. We will do breast pumping alone, as the only standardized means of assessment. This is not an adequate assessment of total milk output, but it will be used as a device to obtain comparison between drugs because it seems to be the easiest procedure to standardize.

Liebrich: Oestrogens would probably affect the fat content of the milk in some way. This is the main source of calories for the baby. The nutritional value of the milk cannot be assessed unless there is some assessment of its quality, perhaps from the rate of weight gain of the baby, to ensure that the right amount of fat is produced and secreted.

Bernard: The weight gain of the infants would be closely observed. The quality of the milk samples would be studied in respect of many constituents, including the free fatty acids and the long-chain fatty acids.

\section{References}

ANON (1976) Lactation, fertility and contraceptives. Lancet, ii, 407.

Buchanan, R. (1975) Breast Feeding: an Aid to Infant Health and Fertility Control. Population

Reports, Series J. 49. George Washington University Medical Center, Washington, DC. 
ChOPRA, J.G. (1972) Effect of steroid contraceptives on lactation. Am J. clin. Nutr. 25, 1202. IPPF (1976) Lactation Research. International Planned Parenthood Federation Medical Bulletin. IPPF, London.

Laumas, K.R., Malkani, P.K. \& BhatnagaR, S. (1967) Radioactivity in the breast milk of lactating women after oral administration of ${ }^{9} \mathrm{H}$-norethynodrel. Am. J. Obstet. Gynec. 98, 411.

Pincus, G., Bialy, G. \& Layne, D.S. (1966) Radioactivity in the milk of subjects receiving radioactive 19-norsteroids. Nature, Lond. 212, 924.

Thomson, A.M., Hytten, F.E. \& BlACK A.E. (1975) Lactation and reproduction. Bull Wld Hlth Org. 52, 337.

VAN DeR MOlen, H.J., HART, P.G. \& WiJMEnGA, H.G. (1969) Studies with $4-^{14}$ C-lynestrenol in normal and lactating women. Acta endocr. 61, 255.

VORHERR, H. (1973) Contraception after abortion and post partum. Am. J. Obstet. Gynec. 117, 1002.

WiJmenga, H.G. \& VAN DER Molen, H.J. (1969) Studies with $4-{ }^{14} \mathrm{C}$-mestranol in lactating women. Acta endocr., Copenh. 61, 665.

WHO (1975) Advances in methods of fertility regulation. Tech. Rep. Ser. Wld Hlth Org. 575, 36.

WHO (1976) Task force on methods for the determination of the fertile period. In: Expanded Programme of Research, Development and Research Training in Human Reproduction, Fifth Annual Report, p. 45. WHO, Geneva. 